WSU-HEP-0808

\title{
Black holes as antimatter factories
}

\author{
Cosimo Bambi ${ }^{1}$, Alexander D. Dolgov ${ }^{2,3,4}$, and Alexey A. Petrov ${ }^{1,5}$ \\ ${ }^{1}$ Department of Physics and Astronomy, Wayne State University, Detroit, MI 48201, USA \\ ${ }^{2}$ Istituto Nazionale di Fisica Nucleare, Sezione di Ferrara, I-44100 Ferrara, Italy \\ ${ }^{3}$ Dipartimento di Fisica, Università degli Studi di Ferrara, I-44100 Ferrara, Italy \\ ${ }^{4}$ Institute of Theoretical and Experimental Physics, 113259 Moscow, Russia \\ ${ }^{5}$ Michigan Center for Theoretical Physics, University of Michigan, Ann Arbor, MI 48109, USA
}

(Dated: November 2, 2018)

\begin{abstract}
We consider accretion of matter onto a low mass black hole surrounded by ionized medium. We show that, because of the higher mobility of protons than electrons, the black hole would acquire positive electric charge. If the black hole's mass is about or below $10^{20} \mathrm{~g}$, the electric field at the horizon can reach the critical value which leads to vacuum instability and electron-positron pair production by the Schwinger mechanism. Since the positrons are ejected by the emergent electric field, while electrons are back-captured, the black hole operates as an antimatter factory which effectively converts protons into positrons.

PACS numbers: 98.62.Mw, 97.60.Lf
\end{abstract}

INTRODUCTION - It is well-known fact that the difference between the mass of proton and electron can lead to predominant capture of protons by celestial bodies, which can make them electrically charged [1]. This process can be exemplified by the case of a cold object, such as a planet, surrounded by an atmosphere of positive ions and electrons in thermal equilibrium. Here, the escape velocity can be reached easier by electrons, which thus would leave the atmosphere. This process leads to the appearance of growing electrostatic field around the planet, making electron escape more and more difficult. The equilibrium is eventually reached when the sums of all forces acting on ions and electrons are equal, which results in non-zero charge acquired by the planet. A similar mechanism can apply to a radiating object. While protons and electrons are gravitationally attracted towards the radiating body with the same acceleration, the outgoing radiation acts differently on the two particle species. Indeed, as the interaction between the outgoing electromagnetic radiation and charged particles is described by the Thompson cross-section, the $e \gamma$ interaction dominates the $p \gamma$ one by a huge factor of $\left(m_{p} / m_{e}\right)^{2} \sim 3 \cdot 10^{6}$, making proton-photon interaction effectively negligible. The stationary solution is again the one in which the astrophysical body is electrically charged, so the emergent electrostatic field makes protons and electrons fall with the same acceleration. In the standard case of radiative atmospheres the effect of the accumulated charge is negligible, so the electric field is not taken into account.

In ref. 2], the authors discussed the case of a black hole (BH) which accreted spherically with luminosity close to the Eddington limit. If the $\mathrm{BH}$ mass is smaller than about $10^{20} \mathrm{~g}$, the electrostatic field of the stationary configuration at horizon exceeds the critical limit to produce electron-positron pairs. The crucial ingredient of the mechanism is that $\mathrm{BH}$ accretion has to be close to the Eddington limit, because the outgoing radiation has to inhibit the electron accretion. This is only possible if the density of the surrounding matter is huge, roughly above $10^{24}$ protons $/ \mathrm{cm}^{3} \approx$ $1 \mathrm{~g} / \mathrm{cm}^{3}$. In this paper we revisit this mechanism, taking the particle thermal distribution into account. We find that the process of proton-to-positron transformation can work even if the particle number density of the medium around the $\mathrm{BH}$ is much lower, close to realistic values. Interesting phenomenological implications are therefore possible.

SCHWINGER MECHANiSM AT SCHWARZSCHILD HORIZON - Let us consider a BH of mass $M$ surrounded by plasma of protons and electrons. In the simplest case of perfect spherical symmetry, the radial part of the equations of motion for the proton and electron fluids are respectively

$$
\begin{aligned}
& \dot{v}_{p}=-\frac{G_{N} M}{r^{2}}+\frac{\alpha Q}{r^{2} m_{p}}+\frac{L \sigma_{\gamma p}}{4 \pi r^{2} m_{p}}-\frac{\sigma_{\gamma p} n_{\gamma} \omega_{\gamma}}{m_{p}} v_{p}-\frac{n_{p} \sigma_{p e} P}{m_{p}}\left(v_{p}-v_{e}\right), \\
& \dot{v}_{e}=-\frac{G_{N} M}{r^{2}}-\frac{\alpha Q}{r^{2} m_{e}}+\frac{L \sigma_{\gamma e}}{4 \pi r^{2} m_{e}}-\frac{\sigma_{\gamma e} n_{\gamma} \omega_{\gamma}}{m_{e}} v_{e}+\frac{n_{e} \sigma_{p e} P}{m_{e}}\left(v_{p}-v_{e}\right) .
\end{aligned}
$$

Here $v_{p}$ and $v_{e}$ are the proton and electron fluid velocities (that is the average velocities of $p$ and $e$ in the plasma which do not include the large chaotic thermal velocities), $Q$ is the electric charge of the $\mathrm{BH}$ in proton charge units, $\alpha=e^{2} / 4 \pi=1 / 137, \sigma_{i j}$ is the cross section of scattering of $i$ on $j, L$ is the luminosity in the comoving frame of the accretion flow, $P$ is the momentum transfer in $e p$-scattering, $n_{p}$ and $n_{e}$ are the number densities of $p$ and $e$ around the $\mathrm{BH}, n_{\gamma}$ is the photon number density in the photon bath surrounding the $\mathrm{BH}$ and $\omega_{\gamma}$ is the photon energy, which is roughly the momentum transfer in $p \gamma-$ and $e \gamma$-scattering. We neglect the angular momentum term because, as explained below, we are interested in the particles with low angular momentum. Eqs. (11) and (2) indeed recover the Eddington limit for $Q=0$ and stationary flux $\dot{v}_{j}=0$ if the fourth term on the right hand side of the two equations, 
that is, the term due to scattering on the thermal bath of photons, is neglected. Taking $Q=0, n_{p}=n_{e}$ and $\dot{v}_{j}=0$, we can solve the two equations for $L$, finding the usual Eddington luminosity, $L_{E}=4 \pi G_{N} M m_{p} / \sigma_{e \gamma}$.

In what follows, it turns out to be useful to define the dimensionless quantities

$$
K=\frac{m_{p}}{m_{e}} \gg 1, \quad q=\frac{\alpha Q}{r_{g} m_{p}} \quad \text { and } \quad l=\frac{L \sigma_{p \gamma}}{4 \pi r_{g} m_{p}} \ll 1,
$$

where $r_{g}=2 G_{N} M$ is the gravitational radius of the $\mathrm{BH}$. Since the cross sections of $e \gamma$ - and $p \gamma$-scattering is inversely proportional respectively to $m_{e}^{2}$ and $m_{p}^{2}$, the impact of the radiation pressure on acceleration for protons is suppressed with respect to electrons by $K^{3}$.

Let us consider particles inside the mean free path from the $\mathrm{BH}, r<\lambda_{j}(j=p, e)$. If their velocity is small enough, i.e. $v_{j}<v_{j}^{c}(r)=\sqrt{F_{j} / r}$, where $F_{p}=r_{g}(1-2 q-2 l)$ and $F_{e}=r_{g}\left(1+2 K q-2 l K^{3}\right)$, such particles would be captured by the BH. Even if we added the angular momentum term into eqs. (11) and (2), which is about $J^{2} / r^{3}$ and has positive sign preventing accretion, the rather small velocities lead to a centrifugal to gravitational force ratio

$$
\frac{J^{2} / r^{3}}{G_{N} M / r^{2}}=\frac{r v_{p}^{2}}{G_{N} M}<\frac{F_{p}}{G_{N} M}=2(1-2 q-2 l)
$$

for protons and $2\left(1+2 K q-2 l K^{3}\right)$ for electrons. Since this ratio for protons is always smaller than 2 , the account of their angular momentum cannot significantly change the protons' accretion, while the electrons' accretion may noticeably slow down. This would increase the charging rate. Then, most of the captured particles are quickly swallowed by the BH. Here indeed the picture is quite different from the widely studied astrophysical accretion for large objects: charged particles can easily lose angular momentum by emitting classical electromagnetic radiation due to accelerating motion around the $\mathrm{BH}$ and probably even by Coulomb scattering, because of the higher particle number density in the vicinity of the $\mathrm{BH}$.

The particles propagate freely inside distance $r$, so we can assume thermal distribution and consider their motion under the influence of gravitational and Coulomb forces only. The number density of particles with velocity $v_{j}<v_{j}^{c}$ is

$$
n_{j}^{c}(r)=C_{j}(r) \int_{0}^{v_{j}^{c}(r)} d v v^{2} \exp \left(-\frac{m_{j} v^{2}}{2 T}\right) .
$$

If the plasma were in equilibrium, $C_{j}(r)$ would be given by $C_{j}(r)=C_{j} \exp \left(G_{N} M m_{j} / r T\right)$, where $C_{j}$ is the value of $C_{j}(r)$ far from the BH. However, in what follows we neglect the dependence of $C_{j}(r)$ on $r$ and we take $C_{j}(r)=C_{j}$ : the $\mathrm{BH}$ accretion is actually determined by the particle distribution far from the $\mathrm{BH}$, where the chemical potential is small, while in the neigborhood of the $\mathrm{BH}$, protons and electrons are not in equilibrium, since there is an intense ingoing flux to the $\mathrm{BH}$. The quantity $C_{j}$ can be determined by the normalization condition

$$
n_{j}=C_{j} \int_{0}^{\infty} d v v^{2} \exp \left(-\frac{m v^{2}}{2 T}\right)=C_{j} \frac{\sqrt{\pi}}{4}\left(\frac{2 T}{m}\right)^{3 / 2},
$$

where $n_{j}$ is the particle number density at infinity and we can reasonably assume that $n_{p}=n_{e}$.

The total number of particles with $v_{j}<v_{j}^{c}$ inside the shell from $r$ to $r+d r$ is $d n_{j}(r)=4 \pi r^{2} n_{j}^{c}(r) d r$. By definition of $n_{j}^{c}, d n_{j}(r)$ provides the number of particles at distance $r$ which would be captured by the BH if they did not collide with other particles in the surrounding plasma. The account of collisions on particle propagation is described by the equation $d n_{j} / d x=-\lambda_{j} n_{\gamma}$, where $\lambda_{j}$ is the particle's mean free path in the medium. In the case of collisions with photons in the thermal bath with temperature $T$, the mean free path is given by

$$
\lambda_{j}=\frac{1}{\sigma_{j \gamma} n_{\gamma}} \sqrt{\frac{m_{j}}{T}}
$$

where $\sigma_{j \gamma}=8 \pi \alpha^{2} / m_{j}^{2}, n_{\gamma}=0.24 T^{3}$ is the number density of photons and the last factor accounts for the necessity to make $\sqrt{m_{j} / T}$ collisions to transfer momentum of the order of the thermal momentum $\sim \sqrt{m_{j} T}$. The latter factor is absent for low momentum particles. Anyhow its presence or absence does not significantly change the results. So, we multiply this quantity by $\exp \left(-r / \lambda_{j}\right)$ to take into account the effects of the scattering in the plasma. To estimate the capture rate we need to divide this quantity by the time of the propagation from $r$ to zero. This time can be found by the integration of the equation of motion $\ddot{r}_{j}=-F_{j} /\left(2 r^{2}\right)$. In principle, the result depends upon the initial velocity 
and direction. However, an order of magnitude estimate for the time is $t(r)=2 / 3 r^{3 / 2} F_{j}^{-1 / 2}$. The total number of captured particles ( $p$ or $e$ ) per unit time is

$$
\begin{aligned}
\dot{N}_{j} & =4 \pi C_{j} \int_{0}^{\infty} d r \frac{r^{2}}{t(r)} \exp \left(-r / \lambda_{j}\right) \int_{0}^{v_{j}^{c}(r)} d v v^{2} \exp \left(-m_{j} v^{2} / 2 T\right) \\
& =24 \sqrt{\pi} n_{j} F_{j}^{2}\left(\frac{m_{j}}{2 T}\right)^{3 / 2} \int_{0}^{\infty} \frac{d x}{x} \exp \left(-r_{g} x / \lambda_{j}\right) \int_{0}^{1} d u u^{2} \exp \left(-\frac{m_{j} u^{2} F_{j}}{2 T r_{g} x}\right) .
\end{aligned}
$$

The main contribution to this integral comes from small $x$, where the exponent in the second term is equal to 1 , so

$$
\dot{N}_{j}=8 \sqrt{\pi} n_{j} F_{j}^{2}\left(\frac{m_{j}}{2 T_{j}}\right)^{3 / 2} \ln \frac{\lambda_{j}}{r_{g}} .
$$

The temperature of protons may be smaller than the temperature of electrons. The latter is usually equal to the temperature of the photon bath. The mean free paths $\lambda_{j}$ of $e$ and $p$ are also different, but their impact on the result is weak, because $\dot{N}_{j}$ depends on $\lambda_{j}$ logarithmically.

The equilibrium state would be reached if $\dot{N}_{p}=\dot{N}_{e}$. Keeping in mind that $F_{p} \approx 1+2 q$ and $F_{e} \approx 1+2 K q$, we find that the equilibrium takes place for

$$
(1+2 q)^{2}\left(m_{p} / 2 T_{p}\right)^{3 / 2} \ln \left(\lambda_{p} / r_{g}\right) \approx(1+2 K q)^{2}\left(m_{e} / 2 T_{e}\right)^{3 / 2} \ln \left(\lambda_{e} / r_{g}\right) .
$$

Let us assume $K q \gg 1 \gg q$. In this case we get

$$
4 K^{2} q^{2} \approx K^{3 / 2}\left(\frac{T_{e}}{T_{p}}\right)^{3 / 2} \frac{\ln \left(\lambda_{p} / r_{g}\right)}{\ln \left(\lambda_{e} / r_{g}\right)} .
$$

Since $\lambda_{p} / \lambda_{e} \approx K^{5 / 2}$ (see eq. (7)), at the temperature $T=1 \mathrm{keV}$ one finds

$$
\frac{\ln \left(\lambda_{p} / r_{g}\right)}{\ln \left(\lambda_{e} / r_{g}\right)}=1+\frac{\ln \left(\lambda_{p} / \lambda_{e}\right)}{\ln \left(\lambda_{e} / r_{g}\right)} \approx 1.7
$$

Thus $K q \sim 100$ and $q \sim 0.1$ and the assumption $K q \gg 1 \gg q$ is consistent. The accumulated electric charge at equilibrium is

$$
\alpha Q \approx \frac{r_{g} m_{p}}{2 K^{1 / 4}}\left(T_{e} / T_{p}\right)^{3 / 4} \sqrt{\frac{\ln \left(\lambda_{p} / r_{g}\right)}{\ln \left(\lambda_{e} / r_{g}\right)}}
$$

and, for $T_{p}=T_{e}$, we obtain $\alpha Q \approx 0.10 m_{p} r_{g}$. The electric charge $Q$, and hence the efficiency of our mechanism, strongly depends upon the temperatures of protons and electrons. We expect that they are different for different astrophysical environments. The case $T_{p}=T_{e}=T_{\gamma}$ is the most reasonable, because the coupling of electrons and protons to the thermal bath of the surrounding photons is strong enough to establish equality of their temperatures. If it were not so, one may argue that $T_{p}$ is larger than $T_{e}$ because the accreting protons have virial temperature, but electrons cool efficiently, so that $T_{p} / T_{e} \gg 1$ and $Q$ is much smaller than our estimate. That does not occur here, because the accretion onto small BHs is quite different from the one onto the Solar-mass BHs. The Eddington accretion for small BHs is typically several orders of magnitude larger than the Bondi accretion, because the former is proportional to the $\mathrm{BH}$ mass, while the latter - to the square of the $\mathrm{BH}$ mass. For example, in the case of $10^{20} \mathrm{~g}$ BH inside a cloud with density $10^{14} \mathrm{~cm}^{-3}$, the Eddington to Bondi accretion rate is about $10^{-10}$. We should thus expect that there are not many collisions between particles of the accreting matter and the gravitational energy is not released efficiently, but advected into the horizon where it is lost. The efficiency parameter $\eta$ is surely very low and $T_{e}$ is close to $T_{p}$. Moreover, it is even more probable that there is no accretion disk at all: the gravitational radius of a $10^{20} \mathrm{~g} \mathrm{BH}$ is about $10^{-8} \mathrm{~cm}$, i.e. the size of an atom. At such small scales, quantum effects are important and they should prevent the formation of a "regular" disk. On the other hand, as already said, most of the captured particles are quickly swallowed by the BH because they emit radiation to their orbit acceleration and thus lose angular momentum efficiently.

If the electric field at the horizon is strong enough, there is the possibility of particle pair production by vacuum breakdown (or Klein instability) [3]. If the $\mathrm{BH}$ radius is much larger than the Compton wavelength of the electron $1 / m_{e}=4 \cdot 10^{-11} \mathrm{~cm}$, i.e. the BH mass is $M \gg 2 \cdot 10^{17} \mathrm{~g}$, we can assume that the electrostatic field near horizon does 
not change with the distance and the electron-positron production proceeds according to the Schwinger result [4] [13]. The pair production probability per unit time and volume is

$$
W=\frac{m_{e}^{4}}{\pi^{2}}\left(\frac{E}{E_{c}}\right)^{2} \sum_{n=1}^{\infty} \frac{1}{n^{2}} \exp \left(-\frac{n \sqrt{\pi} E_{c}}{2 E}\right)
$$

where $E_{c}=m_{e}^{2} / e$. Even if $E \neq 0$ implies $W \neq 0$, the production of particles in a uniform electrostatic field is efficient only if $E$ is close to the critical value $E_{c}$. In our case, the equilibrium electric field at horizon is

$$
E=\frac{\alpha Q}{r_{g}^{2}} \approx 0.10 \frac{m_{p}}{r_{g}}
$$

and $E_{c} / E=0.9\left(M / 10^{20} \mathrm{~g}\right)$. The accretion rate of protons (9) is

$$
\dot{N}_{p}=10^{16} \frac{n_{p}}{10^{10} / \mathrm{cm}^{3}}\left(\frac{r_{g}}{10^{-8} \mathrm{~cm}}\right)^{2}\left(\frac{1 \mathrm{keV}}{T_{p}}\right)^{3 / 2}\left[\frac{\ln \left(\lambda_{p} / r_{g}\right)}{40}\right] \mathrm{s}^{-1}
$$

and since the value of the electric field at horizon is close to $E_{c}$, the $e^{+} e^{-}$pair production is fast and the rate is at the level of the proton accretion rate, that is eq. (16). Then, the created positron would run away from the $\mathrm{BH}$ due to Coulomb repulsion, while electrons are predominantly back-captured: the BH works actually as an efficient antimatter factory, converting protons into positrons. The energy of positrons at infinity is about $\alpha Q / r_{g} \approx 0.15 m_{p} \approx 140 \mathrm{MeV}$.

Discussion - Small BHs with the mass at the level of $10^{20} \mathrm{~g}$ surely cannot be produced by the stellar collapse, but might be created in the early Universe, through many possible mechanisms [ 5 , [6]. Then, if their mass is larger than $5 \cdot 10^{14} \mathrm{~g}$, their lifetime exceeds the age of the Universe and they could still live somewhere today. The proposed mechanism of positron production works if the primordial $\mathrm{BH}$ is surrounded by a ionized medium with the density of roughly $10^{10}$ particles $/ \mathrm{cm}^{3}$, which is about fourteen orders of magnitude smaller than the density required in ref. [2]. For example, the mechanism can operate in the neighborhood of the super-massive BH in the Galactic Center. Here one finds an ionized medium with temperature of roughly $1 \mathrm{keV}$ and particle number density of the order of $10^{8} \mathrm{~cm}^{-3}$ in a sphere of radius $\sim 10^{16} \mathrm{~cm}$ around the central $\mathrm{BH}$ [7]. A more favorable environment is the hot and dense atmosphere around accreting compact stars: in the accretion disk, the temperature of the plasma can reach $10 \mathrm{keV}$ and the particle number density can be as high as $10^{20} \mathrm{~cm}^{-3}[8]$. Primordial BHs may be abundant around "old compact objects", e.g. the final product of the first stars, because captured in the early Universe [9]. They can still live today in the accretion disk of these objects because they cannot be swallowed quickly by their hosts like ordinary matter: they do not lose energy easily. The main mechanism of energy loss for small BHs in a plasma is via dynamical friction and the phenomenon is discussed in ref. [9]. One can see that, for BHs with a mass around $10^{20} \mathrm{~g}$ and for reasonable values of density and temperature of the plasma, the time necessary to fall into the central object exceeds the Universe age.

In principle there are three observable phenomena induced by the process of proton-to-positron transformation.

i) Observation of $\mathrm{MeV}$ positrons in cosmic rays: such positrons would be hard to observe, because they would quickly stop and annihilate in the high density environment. Indeed the mean free path of $100 \mathrm{MeV}$ positrons due to scattering on the photon bath with temperature $T$ is

$$
\lambda_{e}=\frac{1}{\sigma_{e \gamma} n_{\gamma}} \sqrt{\frac{E_{e}}{T}} \sim 10^{5}\left(\frac{1 \mathrm{keV}}{T}\right)^{3} \mathrm{~cm} .
$$

So they stop quite fast and annihilate with electrons. Also, the cross section $e \gamma$ should be smaller than the Thompson one, but for $100 \mathrm{MeV}$ positrons and $1 \mathrm{keV}$ photons the correction is of order one and does not change our conclusions.

ii) Observation of the $0.511 \mathrm{MeV}$ line from $e^{+} e^{-}$annihilation. Here the problem is that the dense medium, where the small $\mathrm{BHs}$ should be to efficiently transform protons into positrons, would disperse this line to a continuos background. Still the line might survive if the effective size of the dense region around the BHs is, at least in one direction, smaller than the photon mean free path. The latter can be estimated as

$$
\lambda_{\gamma}=\frac{1}{\sigma_{e \gamma} n_{e}} \sim 10^{14}\left(\frac{10^{10} \mathrm{~cm}^{-3}}{n_{e}}\right) \mathrm{cm}
$$

for $0.5 \mathrm{MeV}$ photons due to scattering on the background electrons. So, the possibility of observing the line depends crucially on $n_{e}$. It is natural to wonder whether the mechanism can explain the observed $511 \mathrm{keV}$ line from the 
Galactic Center [10], where the expected annihilation rate is at the level of $10^{43} \mathrm{~s}^{-1}$ and several different mechanisms have been already proposed [11]. From eq. (16), we see that the number of BHs should be

$$
N_{B H} \sim 10^{27}\left(\frac{10^{10} \mathrm{~cm}^{-3}}{n_{p}}\right)\left(\frac{10^{20} \mathrm{~g}}{M}\right)^{2}\left(\frac{T_{p}}{1 \mathrm{keV}}\right)^{3 / 2} .
$$

Since the total mass of non-baryonic matter in the Bulge cannot be larger than $10^{10} M_{\odot}[12$, the small BHs have to live in regions with $n_{p}>10^{14} \mathrm{~cm}^{-3}$. It is difficult to make concrete predictions, as the number density of BHs and the temperature and particle number density of the environment are unknown. The upper bound on $N_{B H}$ is given by the condition that their total mass cannot be larger than the maximum mass of dark matter (there are indeed no other constraints for BHs with masses in the range $10^{17}-10^{27} \mathrm{~g}[\underline{6}]$ ). Their distribution would be surely inhomogeneous and it is not unreasonable that they can be accumulated around compact bodies in the center of the Galaxy.

iii) A more realistic possibility is likely the observation of the total gamma ray luminosity created by the annihilated $100 \mathrm{MeV}$ positrons. According to eq. (16), each BH emits about $10^{16} \mathrm{erg} / \mathrm{s}$ for $n_{p}=10^{14} \mathrm{~cm}^{-3}$. If a total amount of $10-100 M_{\odot}$ small BHs are accumulated into a high density cloud/atmosphere, they may be observable as a luminous object with the power of about $10^{30}-10^{31} \mathrm{erg} / \mathrm{s}$.

CONCLUSION - If primordial light black holes surrounded by a ionized medium exist in the Galaxy, they could operate as an efficient antimatter factories converting accreting protons into positrons. The charging of $\mathrm{BH}$ by the predominant influx of protons in comparison to electrons is achieved because of the much larger proton mass and correspondingly much larger proton mobility in the surrounding medium. If the black hole is small, the electrostatic field at the horizon can exceed the critical value of the vacuum stability and electron-positron pair production by Schwinger mechanism becomes efficient. Primordial black holes with the mass in the range $10^{15}-10^{20} \mathrm{~g}$ might live today in the Universe, injecting positrons. If these black holes live in an ionized high density medium, such as around the super-massive black hole in the Galactic Center or in the atmosphere of violent stars, the proton conversion into energetic positrons would enrich cosmic rays by positrons with energeis in the interval 1-100 MeV and gamma radiation of similar in energies, which possibly makes the effect experimentally observable.

ACKNOWLEDGments - C.B. and A.A.P. are supported in part by NSF under grant PHY-0547794 and by DOE under contract DE-FG02-96ER41005.

[1] V.F. Shvartsman, Astrofizika 6, 309 (1970); Ya.B. Zeldovich and I.D. Novikov, Relativistic Astrophysics. Vol. 1: Stars and Relativity (Chicago University Press, Chicago, USA, 1971); A.M. Anile and A. Treves, Astrophys. Space Sc. 19, 411 (1972); R. Turolla, S. Zane, A. Treves and A. Illarionov, Astrophys. J. 482, 377 (1997).

[2] A. Treves and R. Turolla, Astrophys. J. 517, 396 (1999).

[3] G.W. Gibbons, Commun. Math. Phys. 44, 245 (1975); T. Damour and R. Ruffini, Phys. Rev. Lett. 35, 463 (1975); G. Preparata, R. Ruffini and S.S. Xue, Astron. Astrophys. 338, L87 (1998); I.B. Khriplovich, J. Exp. Theor. Phys. 88, 845 (1999) [Zh. Eksp. Teor. Fiz. 115, 1539 (1999)].

[4] F. Sauter, Z. Phys. 69, 742 (1931); J.S. Schwinger, Phys. Rev. 82, 664 (1951).

[5] A. Dolgov and J. Silk, Phys. Rev. D 47, 4244 (1993); A.D. Dolgov, M. Kawasaki and N. Kevlishvili, Nucl. Phys. B (in press) arXiv:0806.2986 [hep-ph]].

[6] B.J. Carr, Lect. Notes Phys. 631, 301 (2003) arXiv:astro-ph/0310838.

[7] F. Melia and H. Falcke, Ann. Rev. Astron. Astrophys. 39, 309 (2001) arXiv:astro-ph/0106162.

[8] S.L. Saphiro and S.A. Teukolsky, Black Holes, White Dwarfs, and Neutron Stars (John Wiley, New York, USA, 1983).

[9] C. Bambi, D. Spolyar, A.D. Dolgov, K. Freese and M. Volonteri, MNRAS (in press) arXiv:0812.0585 [astro-ph]].

[10] J. Knodlseder et al., Astron. Astrophys. 441, 513 (2005) arXiv:astro-ph/0506026].

[11] P.A. Milne, L.S. The and M.D. Leising, Astrophys. J. Suppl. 124, 503 (1999); N. Guessoum, P. Jean and N. Prantzos, Astron. Astrophys. 457, 753 (2006); C. Boehm et al, Phys. Rev. Lett. 92, 101301 (2004); C. Picciotto and M. Pospelov, Phys. Lett. B 605, 15 (2005); D.H. Oaknin and A.R. Zhitnitsky, Phys. Rev. Lett. 94, 101301 (2005); C. Bambi and A.D. Dolgov, Nucl. Phys. B 784, 132 (2007); C. Bambi, A.D. Dolgov and A.A. Petrov, Phys. Lett. B 670, 174 (2008).

[12] R. Schaeffer, D. Méra and G. Chabrier, Acta Physica Pol. B 29, 1905 (1998).

[13] If this were not so, we would have to take into account a decrease of the field at the Compton wave length of electron. This would lead to larger value of the critical field, see the last paper in ref. [3] . 